\title{
Power Control Games Between Anchor and Jammer Nodes in Wireless Localization Networks
}

\author{
Ahmet Dundar Sezer ${ }^{(0)}$, Student Member, IEEE and Sinan Gezici ${ }^{(0)}$, Senior Member, IEEE
}

\begin{abstract}
In this paper, a game theoretic framework is proposed for wireless localization networks that operate in the presence of jammer nodes. In particular, power control games between anchor and jammer nodes are designed for a wireless localization network in which each target node estimates its position based on received signals from anchor nodes while jammer nodes aim to reduce localization performance of target nodes. Two different games are formulated for the considered wireless localization network: In the first game, the average Cramér-Rao lower bound (CRLB) of the target nodes is considered as the performance metric, and it is shown that at least one pure strategy Nash equilibrium exists in the power control game. Also, a method is presented to identify the pure strategy Nash equilibrium, and a sufficient condition is obtained to resolve the uniqueness of the pure Nash equilibrium. In the second game, the worst-case CRLBs for the anchor and jammer nodes are considered, and it is shown that the game admits at least one pure Nash equilibrium. Numerical examples are presented to corroborate the theoretical results.
\end{abstract}

Index Terms-Localization, jammer, power allocation, Nash equilibrium, estimation, wireless network.

\section{INTRODUCTION}

I N RECENT years, research communities have developed a significant interest in wireless localization networks, which provide important applications for various systems and services [1], [2]. To name a few, smart inventory tracking systems, location sensitive billing services, and intelligent autonomous transport systems benefit from wireless localization networks [3]. In such a wide variety of applications, accurate and robust position estimation plays a crucial role in terms of efficiency and reliability. In the literature, various theoretical and experimental studies have been conducted in order to analyze wireless position estimation in the context of accuracy requirements and system constraints; e.g., [4], [5].

In a wireless localization network, there exist two types of nodes in general; namely, anchor nodes and target nodes. Anchor nodes have known positions and their location information is available at target nodes. On the other hand, target nodes have unknown positions, and each target node in the network

Manuscript received July 3, 2017; revised November 9, 2017; accepted January 5, 2018. Date of publication January 23, 2018; date of current version August 7, 2018. The work of A. D. Sezer was supported by ASELSAN Graduate Scholarship for Turkish Academicians. The associate editor coordinating the review of this manuscript and approving it for publication was Prof. Michael Rabbat. (Corresponding author: Sinan Gezici.)

The authors are with the Department of Electrical and Electronics Engineering, Bilkent University, Ankara 06800, Turkey (e-mail: adsezer@ee. bilkent.edu.tr; gezici@ee.bilkent.edu.tr).

Digital Object Identifier 10.1109/TSIPN.2018.2797020 estimates its own position based on received signals from anchor nodes (in the case of self localization [3]). In particular, position estimation of a target node is performed by using various signal parameters extracted from received signals (i.e., waveforms). Commonly employed signal parameters are time-ofarrival (TOA) [6], [7], time-difference-of-arrival (TDOA) [8], angle-of-arrival (AOA) [9], and received signal strength (RSS) [10]. TOA and TDOA are time based parameters which measure the signal propagation time (difference) between nodes. AOA is obtained based on the angle at which the transmitted signal from one node arrives at another node. RSS is another signal parameter which gathers information from power or energy of a signal that travels between anchor and target nodes [4]. Since a signal traveling from an anchor node to a target node experiences multipath fading, shadowing, and path-loss, position estimates of target nodes are subject to errors and uncertainty. As the Cramér-Rao lower bound (CRLB) expresses a lower bound on the variance of any unbiased estimator for a deterministic parameter, it is also considered as a common performance metric for wireless localization networks [11]-[13].

Besides anchor and target nodes, a wireless localization network can contain undesirable jammer nodes, the aim of which is to degrade the localization performance (i.e., accuracy) of the network. In the literature, various studies have been performed on the jamming of wireless localization networks. The jamming and anti-jamming of the global positioning system (GPS) are studied in [14] for various jamming schemes. Similarly, in [15], an adaptive GPS anti-jamming algorithm is proposed. In addition, the optimal power allocation problem is investigated for jammer nodes in a given wireless localization network based on the CRLB metric, and the optimal jamming strategies are obtained in the presence of peak power and total power constraints in [11].

In the literature, various studies have been conducted on power allocation for wireless localization networks [16]-[19]. In [16], the optimal anchor power allocation strategies are investigated together with anchor selection and anchor deployment strategies for the minimization of the squared position error bound (SPEB), which identifies fundamental limits on localization accuracy. The work in [17] provides a robust power allocation framework for network localization in the presence of imperfect knowledge of network parameters. Based on the performance metrics SPEB and the directional position error bound (DPEB), the optimal power allocation problems are formulated in the consideration of limited power resources and it is shown that the proposed problems can be solved via conic 
programming. In [18], ranging energy optimization problems are investigated for an unsynchronized positioning network based on two-way ranging between a sensor and beacons. In [19], the work in [18] is extended for a positioning network in which the collaborative anchors added to the system help sensors locate themselves.

In the presence of jammer nodes in a wireless localization network, anchor nodes can adapt their power allocation strategies in response to the strategies employed by jammer nodes and enhance the localization performance of the network. On the other hand, jammer nodes can respond by updating their corresponding power allocation strategies in order to degrade the localization performance. These conflicting interests between anchor and jammer nodes can be analyzed by employing game theory as a tool. In the literature, game theoretic frameworks have been applied for investigating power allocation strategies of users in a competitive system. In [20], competitive interactions between a secondary user transmitter-receiver pair and a jammer are analyzed by applying a game-theoretic framework in the presence of interference constraints, power constraints, and incomplete channel gain information. In particular, the strategic power allocation game between the two players is proposed first, and then it is presented that the solution of the game corresponds to Nash equilibria points. In [21], a zero-sum game is modeled between a centralized detection network and a jammer in the presence of complete information. It is obtained that the jammer has no effect on the error probability observed at the fusion center when it employs pure strategies at the Nash equilibrium.

Although there exist research papers that analyze the noncooperative behavior of system users and jammer nodes in wireless communication networks in terms of successful transmissions under a minimum signal-to-interference-plus-noise ratio (SINR) constraint and error probability [20], [21], no studies in the literature have investigated the interactions between anchor nodes and jammer nodes in a wireless localization network, where target nodes estimate their positions based on signals received from anchor nodes and jammer nodes try to degrade the localization performance of the network. In the field of wireless localization, there exist some recent studies (e.g., [13] and [22]) that analyze the interactions of entities in a wireless localization network. However, no jammer nodes are considered in those studies, which focus on a cooperative localization network where the target nodes share information with each other to improve their position estimates. Therefore, the theoretical analyses presented therein differ from the ones performed in this paper, which considers non-cooperative localization where anchor and jammer nodes compete for the localization performance of target nodes.

In this paper, power control games between anchor and jammer nodes are designed based on a game-theoretic framework by employing the CRLB metric. In particular, two different games are formulated for the considered wireless localization network: In the first game, the average CRLB of the target nodes is considered as the performance metric whereas in the second one, the worst-case CRLBs for the anchor and jammer nodes are employed. As a solution approach, Nash equilibria of the games are examined, and it is shown that a pure Nash equilibrium exists in both of the proposed power control games. In addition, for the game in which the anchor and jammer nodes compete according to the average CRLB, a method is presented to obtain a pure strategy Nash equilibrium and a sufficient condition is provided to decide whether the pure strategy Nash equilibrium is unique. Finally, numerical examples are presented to demonstrate the theoretical results.

The main contributions of this work can be summarized as follows:

- A game theoretic formulation is developed between anchor and jammer nodes in a wireless localization network for the first time in the literature.

- Two types of power control games between anchor and jammer nodes are proposed based on the average CRLB and the worst-case CRLBs for the anchor and jammer nodes.

- In a game-theoretic framework, the Nash equilibria of the proposed games are analyzed and it is shown that both of the games have at least one pure strategy Nash equilibrium.

- For the game that employs the average CRLB as a performance metric, an approach is developed to obtain the pure strategy Nash equilibrium and a sufficient condition is derived to determine whether the obtained Nash equilibrium is a unique pure strategy Nash equilibrium.

The remainder of the paper is organized as follows: Section II describes the wireless localization network and introduces the network parameters. Section III first presents the proposed game formulations, and then provides detailed theoretical analyses. Numerical results are described in Section IV, which is followed by the concluding remarks in Section V.

\section{SYSTEM MODEL}

Consider a wireless localization network with $N_{A}$ anchor nodes and $N_{T}$ target nodes at locations $\boldsymbol{y}_{i} \in \mathbb{R}^{2}$ for $i \in$ $\left\{1, \ldots, N_{A}\right\}$ and $\boldsymbol{x}_{i} \in \mathbb{R}^{2}$ for $i \in\left\{1, \ldots, N_{T}\right\}$, respectively. Each target node in the system estimates its position based on received signals from the anchor nodes, the locations of which are known by the target nodes (i.e., the target nodes perform self-positioning [3]). Besides the anchor and target nodes, there exist $N_{J}$ jammer nodes located at $\boldsymbol{z}_{i} \in \mathbb{R}^{2}$ for $i \in\left\{1, \ldots, N_{J}\right\}$ in the system. Contrary to the anchor nodes, the aim of the jammer nodes is to reduce the localization performance of the target nodes. In accordance with the common approach in the literature [11], [23]-[25], it is assumed that the jammer nodes transmit zero-mean white Gaussian noise in order to distort the signals observed by the target nodes. The reasons behind the use of a Gaussian noise model can be explained as follows: In wireless localization systems, when the knowledge of the ranging signals sent from the anchor nodes to the target nodes is unavailable to the jammer nodes, the jammer nodes can continuously transmit noise to degrade the localization performance of the target nodes [11]. In the literature, it is shown that the Gaussian noise is the worst-case noise for generic wireless networks modeled with additive noise that is independent of the transmit signals [26]-[28]. (In particular, the Gaussian distribution corresponds to the worst-case scenario among all possible 
noise distributions in terms of some metrics such as the mutual information and the mean squared error since it minimizes the mutual information between the input and the output when the input is Gaussian, and maximizes the mean squared error of estimating the input given the output for an additive noise channel with a Gaussian input [29].) Therefore, the jammer nodes are expected to transmit Gaussian noise for efficient jamming [11]. Also, a non-cooperative localization scenario is considered; that is, the target nodes do not receive any signals from each other for localization purposes.

Let $\mathcal{A}_{i}$ denote the connectivity set for target node $i$, which is defined as $\mathcal{A}_{i} \triangleq\left\{j \in\left\{1, \ldots, N_{A}\right\} \mid\right.$ anchor node $j$ is connected to target node $i\}$ for $i \in\left\{1, \ldots, N_{T}\right\}$. Then, corresponding to the transmission from anchor node $j$, the received signal at target node $i$ can be expressed as

$r_{i j}(t)=\sum_{k=1}^{L_{i j}} \alpha_{i j}^{k} \sqrt{P_{i j}^{A}} s\left(t-\tau_{i j}^{k}\right)+\sum_{l=1}^{N_{J}} \gamma_{i l} \sqrt{P_{l}^{J}} \nu_{i l j}(t)+n_{i j}(t)$

for $t \in\left[0, T_{\text {obs }}\right], i \in\left\{1, \ldots, N_{T}\right\}$, and $j \in \mathcal{A}_{i}$, where $T_{\text {obs }}$ is the observation time, $L_{i j}$ is the number of paths between anchor node $j$ and target node $i, \alpha_{i j}^{k}$ and $\tau_{i j}^{k}$ represent, respectively, the amplitude and the delay of the $k$ th multipath component between anchor node $j$ and target node $i, P_{i j}^{A}$ is the transmit power of the signal sent from anchor node $j$ to target node $i$, and $\gamma_{i l}$ represents the channel coefficient between jammer node $l$ and target node $i$, which has a transmit power of $P_{l}^{J}$ [11]. Also, during the reception from anchor node $j, n_{i j}(t)$ denotes the measurement noise at target node $i$ and $\nu_{i l j}(t)$ represents the jammer noise at target node $i$ generated by jammer node $l$. It is assumed that the transmit signal $s(t)$ is a known signal with unit energy, and the measurement noise $n_{i j}(t)$ and the jammer noise $\nu_{i l j}(t)$ are independent zero-mean white Gaussian random processes, where the spectral density levels of $n_{i j}(t)$ and $\nu_{i l}(t)$ are equal to $N_{0} / 2$ and one, respectively [11]. In addition, for each target node, $n_{i j}(t)$ 's are independent for $j \in$ $\mathcal{A}_{i}$, and $v_{i l j}(t)$ 's are independent for $l \in\left\{1, \ldots, N_{J}\right\}$ and $j \in$ $\mathcal{A}_{i} \cdot{ }^{1}$ The delay $\tau_{i j}^{k}$ is expressed as $\tau_{i j}^{k} \triangleq\left(\left\|\boldsymbol{y}_{j}-\boldsymbol{x}_{i}\right\|+b_{i j}^{k}\right) / c$, where $b_{i j}^{k}$ denotes the non-negative range bias and $c$ is the speed of propagation.

\section{Power Control Games Between ANCHOR AND JAMMER NODES}

In this section, the aim is to design and analyze power control games between anchor and jammer nodes. In the proposed setting, the anchor nodes set their power levels in order to maximize the localization performance of the target nodes whereas the jammer nodes try to minimize the localization performance via power allocation. The localization performance is quantified by the average CRLB for the target nodes, which is the metric according to which the anchor and jammer nodes compete. In

\footnotetext{
${ }^{1}$ As in [11], it is assumed that the anchor nodes transmit at different time intervals to prevent interference at the target nodes [4], and during those time intervals, the channel coefficient between a jammer node and a target node is assumed to be constant.
}

other words, the anchor nodes (jammer nodes) try to minimize (maximize) the average CRLB for the target nodes to improve (deteriorate) the localization performance of the system. The use of the CRLB as the performance metric can be justified based on the following arguments: As investigated in [30], the ML location estimator becomes asymptotically unbiased and efficient for sufficiently large SNRs and/or effective bandwidths, and consequently, it achieves a mean-squared error (MSE) close to the CRLB. For other cases, the CRLB may not provide a tight bound for MSEs of ML estimators [31], [32]. Therefore, the CRLBs obtained based on the optimal power strategies of the anchor and jammer nodes provide performance bounds for the MSEs of the target nodes. Another reason for the use of the CRLB metric is that it leads to compact closed form expressions for the optimization problems and consequently facilitates theoretical analyses, which lead to intuitive explanations of power control games between anchor and jammer nodes. (Performance optimization based on the CRLB has been considered in various studies in the literature such as [11], [13], [33].)

To obtain the formulation of the proposed problem, the CRLB expression for the target nodes is presented as a utility function first, and then the game model is proposed.

\section{A. CRLB for Location Estimation of Target Nodes}

To provide the CRLB expression for target node $i$, the unknown parameters related to target node $i$ are defined as [11]

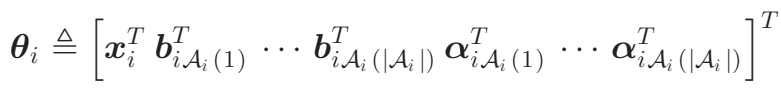

where $\mathcal{A}_{i}(j)$ represents the $j$ th element of set $\mathcal{A}_{i},\left|\mathcal{A}_{i}\right|$ denotes the cardinality of set $\mathcal{A}_{i}, \boldsymbol{\alpha}_{i j}=\left[\alpha_{i j}^{1} \cdots \alpha_{i j}^{L_{i j}}\right]^{T}$, and $\boldsymbol{b}_{i j}$ is defined as

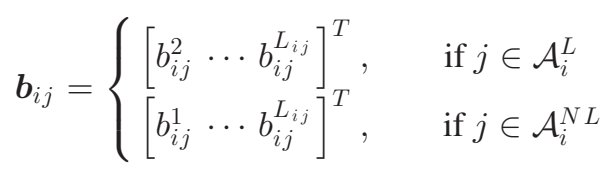

with $\mathcal{A}_{i}^{L}$ and $\mathcal{A}_{i}^{N L}$ representing the sets of anchors nodes that are in the line-of-sight (LOS) and non-line-of-sight (NLOS) of target node $i$, respectively [11]. Then, the CRLB for estimating the location of target node $i$ is given by

$$
\mathbb{E}\left\{\left\|\hat{\boldsymbol{x}}_{i}-\boldsymbol{x}_{i}\right\|^{2}\right\} \geq \operatorname{tr}\left\{\left[\boldsymbol{F}_{i}^{-1}\right]_{2 \times 2}\right\} \triangleq \mathrm{CRLB}_{i}
$$

where $\hat{\boldsymbol{x}}_{i}$ denotes an unbiased estimate of the location of target node $i$, tr represents the trace operator, and $\boldsymbol{F}_{i}$ is the Fisher information matrix for vector $\boldsymbol{\theta}_{i}$ in (2). From [4] and [11], $\left[\boldsymbol{F}_{i}^{-1}\right]_{2 \times 2}$ can be expressed as

$$
\left[\boldsymbol{F}_{i}^{-1}\right]_{2 \times 2}=\boldsymbol{J}_{i}\left(\boldsymbol{x}_{i}, \boldsymbol{p}_{i}^{A}, \boldsymbol{p}^{J}\right)^{-1}
$$

where $\boldsymbol{J}_{i}\left(\boldsymbol{x}_{i}, \boldsymbol{p}_{i}^{A}, \boldsymbol{p}^{J}\right)$ denotes the equivalent Fisher information matrix, which is calculated as

$$
\boldsymbol{J}_{i}\left(\boldsymbol{x}_{i}, \boldsymbol{p}_{i}^{A}, \boldsymbol{p}^{J}\right)=\sum_{j \in \mathcal{A}_{i}^{L}} \frac{P_{i j}^{A} \lambda_{i j}}{N_{0} / 2+\boldsymbol{a}_{i}^{T} \boldsymbol{p}^{J}} \boldsymbol{\phi}_{i j} \boldsymbol{\phi}_{i j}^{T}
$$


with

$$
\begin{aligned}
\lambda_{i j} & \triangleq \frac{4 \pi^{2}\left|\alpha_{i j}^{1}\right|^{2} \int_{-\infty}^{\infty} f^{2}|S(f)|^{2} d f}{c^{2}}\left(1-\xi_{j}\right), \\
\boldsymbol{a}_{i} & \triangleq\left[\begin{array}{lll}
\left|\gamma_{i 1}\right|^{2} \cdots & \left|\gamma_{i N_{J}}\right|^{2}
\end{array}\right]^{T}, \\
\boldsymbol{p}_{i}^{A} & \triangleq\left[\begin{array}{lll}
P_{i \mathcal{A}_{i}(1)}^{A} & \cdots & P_{i \mathcal{A}_{i}\left(\left|\mathcal{A}_{i}\right|\right)}^{A}
\end{array}\right]^{T}, \\
\boldsymbol{p}^{J} & \triangleq\left[\begin{array}{lll}
P_{1}^{J} & \cdots & P_{N_{J}}^{J}
\end{array}\right]^{T} \\
\boldsymbol{\phi}_{i j} & \triangleq\left[\begin{array}{lll}
\cos \varphi_{i j} & \sin \varphi_{i j}
\end{array}\right]^{T} .
\end{aligned}
$$

In (7), $S(f)$ denotes the Fourier transform of $s(t)$, and the pathoverlap coefficient $\xi_{j}$ is a number that satisfies $0 \leq \xi_{j} \leq 1$ [17]. Also, in (11), $\varphi_{i j}$ corresponds to the angle between target node $i$ and anchor node $j$.

\section{B. Power Control Game Model}

Let $\mathcal{G}=\left\langle\mathcal{N},\left(S_{i}\right)_{i \in \mathcal{N}},\left(u_{i}\right)_{i \in \mathcal{N}}\right\rangle$ denote the power control game between anchor nodes (i.e., Player A) and jammer nodes (i.e., Player J), where $\mathcal{N}=\{A, J\}$ is the index set for the players, $S_{i}$ is the strategy set for player $i$, and $u_{i}$ is the utility function of player $i$. For the anchor nodes, strategy set $S_{A}$ is defined as

$$
\begin{array}{r}
S_{A} \triangleq\left\{\boldsymbol{p}^{A} \in \mathbb{R}^{K} \mid \mathbf{1}^{T} \boldsymbol{p}^{A} \leq P_{T}^{A} \wedge 0 \leq \boldsymbol{e}_{i}^{T} \boldsymbol{p}^{A} \leq P_{\text {peak }}^{A},\right. \\
\forall i \in\{1, \ldots, K\}\}
\end{array}
$$

with

$$
\boldsymbol{p}^{A} \triangleq\left[\left(\boldsymbol{p}_{1}^{A}\right)^{T} \cdots\left(\boldsymbol{p}_{N_{T}}^{A}\right)^{T}\right]^{T}
$$

where $\boldsymbol{p}_{i}^{A}$ is as defined in (9), $\mathbf{1}$ is the vector of ones, $\boldsymbol{e}_{i}$ is the unit vector whose $i$ th element is one, $K$ is the dimension of $\boldsymbol{p}^{A}$, $P_{T}^{A}$ is the total available power of the anchor nodes, and $P_{\text {peak }}^{A}$ is the maximum allowed and attainable power (peak power) for the anchor nodes. Similarly, strategy set $S_{J}$ for the jammer nodes is defined as

$$
\begin{array}{r}
S_{J} \triangleq\left\{\boldsymbol{p}^{J} \in \mathbb{R}^{N_{J}} \mid \mathbf{1}^{T} \boldsymbol{p}^{J} \leq P_{T}^{J} \wedge 0 \leq \boldsymbol{e}_{i}^{T} \boldsymbol{p}^{J} \leq P_{\text {peak }}^{J},\right. \\
\left.\forall i \in\left\{1, \ldots, N_{J}\right\}\right\}
\end{array}
$$

where $\boldsymbol{p}^{J}$ is as defined in (10), $P_{T}^{J}$ is the total available power of the jammer nodes, and $P_{\text {peak }}^{J}$ is the maximum allowed and attainable power (peak power) for the jammer nodes.

Let $\boldsymbol{p}^{A}$ and $\boldsymbol{p}^{J}$ denote strategies of player $A$ and player $J$, respectively. Then, a strategy (action) profile of the game can be denoted as $\left(\boldsymbol{p}^{A}, \boldsymbol{p}^{J}\right) \in S$, where $\boldsymbol{p}^{A} \in S_{A}, \boldsymbol{p}^{J} \in S_{J}$, and $S=S_{A} \times S_{J}$. For a given action profile, the utility functions of player $A$ and player $J$ are defined as

$$
\begin{aligned}
& u_{A}\left(\boldsymbol{p}^{A}, \boldsymbol{p}^{J}\right)=-\frac{1}{N_{T}} \sum_{i=1}^{N_{T}} \operatorname{tr}\left\{\boldsymbol{J}_{i}\left(\boldsymbol{x}_{i}, \boldsymbol{p}_{i}^{A}, \boldsymbol{p}^{J}\right)^{-1}\right\}, \\
& u_{J}\left(\boldsymbol{p}^{A}, \boldsymbol{p}^{J}\right)=\frac{1}{N_{T}} \sum_{i=1}^{N_{T}} \operatorname{tr}\left\{\boldsymbol{J}_{i}\left(\boldsymbol{x}_{i}, \boldsymbol{p}_{i}^{A}, \boldsymbol{p}^{J}\right)^{-1}\right\} .
\end{aligned}
$$

Namely, the average CRLB of the target nodes is employed in the utility functions (see (4) and (5)). Since $u_{A}\left(\boldsymbol{p}^{A}, \boldsymbol{p}^{J}\right)$ and $u_{J}\left(\boldsymbol{p}^{A}, \boldsymbol{p}^{J}\right)$ satisfy that $u_{A}\left(\boldsymbol{p}^{A}, \boldsymbol{p}^{J}\right)+u_{J}\left(\boldsymbol{p}^{A}, \boldsymbol{p}^{J}\right)=0 \forall \boldsymbol{p}^{A} \in$ $S_{A} \wedge \forall \boldsymbol{p}^{J} \in S_{J}$, it is noted that the power control game between player $A$ and player $J$ corresponds to a two-player zero-sum game.

\section{Nash Equilibrium in Power Control Game}

The Nash equilibrium is one of the solution approaches that is commonly used for game theoretic problems [34]. In the game-theoretic notation, a strategy profile of game $\mathcal{G}$, denoted as $\left(\boldsymbol{p}_{\star}^{A}, \boldsymbol{p}_{\star}^{J}\right)$, is a Nash equilibrium if

$$
\begin{aligned}
& u_{A}\left(\boldsymbol{p}_{\star}^{A}, \boldsymbol{p}_{\star}^{J}\right) \geq u_{A}\left(\boldsymbol{p}^{A}, \boldsymbol{p}_{\star}^{J}\right), \forall \boldsymbol{p}^{A} \in S_{A}, \\
& u_{J}\left(\boldsymbol{p}_{\star}^{A}, \boldsymbol{p}_{\star}^{J}\right) \geq u_{J}\left(\boldsymbol{p}_{\star}^{A}, \boldsymbol{p}^{J}\right), \forall \boldsymbol{p}^{J} \in S_{J} .
\end{aligned}
$$

At a Nash equilibrium, no player can improve its utility by changing its strategy unilaterally. In other words, given the power levels of player $J$ (player $A$ ), player $A$ (player $J$ ) does not have any incentive to deviate from its power strategy at a Nash equilibrium. Such an equilibrium does not necessarily exist in infinite games. However, power control game $\mathcal{G}$ admits a pure Nash equilibrium as the following proposition states.

Proposition 1: A pure Nash equilibrium exists in power control game $\mathcal{G}$.

Proof: The aim in the proof is to show that the game has at least one pure-strategy Nash equilibrium. For that reason, it is first noted that power control game $\mathcal{G}$ in strategic form $\left\langle\mathcal{N},\left(S_{i}\right)_{i \in \mathcal{N}},\left(u_{i}\right)_{i \in \mathcal{N}}\right\rangle$ admits at least one pure Nash equilibrium if the following conditions are satisfied [35]:

- Strategy set $S_{i}$ is compact and convex for all $i \in \mathcal{N}$, where $\mathcal{N}=\{A, J\}$.

- $u_{i}\left(\boldsymbol{p}^{A}, \boldsymbol{p}^{J}\right)$ is a continuous function in the profile of strategies $\left(\boldsymbol{p}^{A}, \boldsymbol{p}^{J}\right) \in S$ for all $i \in \mathcal{N}$.

- $u_{A}\left(\boldsymbol{p}^{A}, \boldsymbol{p}^{J}\right)$ and $u_{J}\left(\boldsymbol{p}^{A}, \boldsymbol{p}^{J}\right)$ are quasi-concave functions in $\boldsymbol{p}^{A}$ and $\boldsymbol{p}^{J}$, respectively.

Since set $S_{A}$ in (12) and set $S_{J}$ in (14) are closed and bounded, it can easily be shown that the sets in (12) and (14) are compact and convex, which satisfies the first condition. Also, $u_{A}\left(\boldsymbol{p}^{A}, \boldsymbol{p}^{J}\right)$ in (15) is a concave function of $\boldsymbol{p}^{A}$ based on the proof in [36] and $u_{J}\left(\boldsymbol{p}^{A}, \boldsymbol{p}^{J}\right)$ in (16) is a linear (and concave) function of $p^{J}$ based on [33]. Consequently, (15) and (16) are continuous and quasi-concave functions, for which the second and the third conditions hold. Therefore, it is concluded that at least one Nash equilibrium exists in power control game $\mathcal{G}$.

Based on Proposition 1, the proposed power control game has at least one Nash equilibrium. In order to analyze the Nash equilibrium, first, best response strategies of player $A$ and $J$ are discussed and then, a fixed point equation is obtained.

For a given power strategy of player $J$ (i.e., power levels of jammer nodes), the best response function of player $A$ can be expressed as

$$
\begin{aligned}
\boldsymbol{p}_{\mathrm{BR}}^{A} & =\mathrm{BR}_{A}\left(\boldsymbol{p}^{J}\right) \\
& \triangleq \underset{\boldsymbol{p}^{A} \in S_{A}}{\arg \max }-\frac{1}{N_{T}} \sum_{i=1}^{N_{T}} \operatorname{tr}\left\{\boldsymbol{J}_{i}\left(\boldsymbol{x}_{i}, \boldsymbol{p}_{i}^{A}, \boldsymbol{p}^{J}\right)^{-1}\right\} .
\end{aligned}
$$


On the other hand, for a given power strategy of player $A$, the best response function of player $J$ is given as

$$
\begin{aligned}
& \boldsymbol{p}_{\mathrm{BR}}^{J}=\mathrm{BR}_{J}\left(\boldsymbol{p}^{A}\right) \\
& \triangleq \underset{\boldsymbol{p}^{J} \in S_{J}}{\arg \max } \frac{1}{N_{T}} \sum_{i=1}^{N_{T}} \operatorname{tr}\left\{\boldsymbol{J}_{i}\left(\boldsymbol{x}_{i}, \boldsymbol{p}_{i}^{A}, \boldsymbol{p}^{J}\right)^{-1}\right\} .
\end{aligned}
$$

Let $\mathrm{BR}=\left(\mathrm{BR}_{A}, \mathrm{BR}_{J}\right): S=S_{A} \times S_{J} \rightarrow S$ be a mapping of a function (correspondence) $B R(\boldsymbol{p})$, where $\boldsymbol{p}=\left(\boldsymbol{p}^{A}, \boldsymbol{p}^{J}\right) \in S$ is a strategy profile of the power control game, and $\mathrm{BR}_{\mathrm{A}}$ and $\mathrm{BR}_{\mathrm{J}}$ are as in (19) and (20), respectively. Based on the definition of the Nash equilibrium, the following fixed point equation holds for the Nash equilibrium:

$$
\boldsymbol{p}^{\star}=\operatorname{BR}\left(\boldsymbol{p}^{\star}\right)
$$

In addition, the utility function in (15) is a concave function of $\boldsymbol{p}^{A}$ and the utility function in (16) is a linear (and concave) function of $\boldsymbol{p}^{J}$. Based on the utility functions in (15) and (16), the game between player $A$ and player $J$ is called convexconcave game [37], [38]. In a convex-concave game, the Nash equilibrium becomes the saddle-point equilibrium, and if there exist multiple Nash equilibria, the value of the game is unique for every Nash equilibrium. Therefore, the pure Nash equilibrium of power control game $\mathcal{G}$ can be obtained as stated in the following proposition.

Proposition 2: Let $\boldsymbol{p}^{\star}=\left(\boldsymbol{p}_{\star}^{A}, \boldsymbol{p}_{\star}^{J}\right)$ denote the Nash equilibrium of power control game $\mathcal{G}$ in pure strategies. Then, $\boldsymbol{p}^{\star}$ satisfies the following equation:

$$
\begin{aligned}
& u_{J}\left(\boldsymbol{p}_{\star}^{A}, \boldsymbol{p}_{\star}^{J}\right)=-u_{A}\left(\boldsymbol{p}_{\star}^{A}, \boldsymbol{p}_{\star}^{J}\right) \\
& =\min _{\boldsymbol{p}^{A} \in S_{A}} \max _{\boldsymbol{p}^{J} \in S_{J}} \frac{1}{N_{T}} \sum_{i=1}^{N_{T}} \operatorname{tr}\left\{\boldsymbol{J}_{i}\left(\boldsymbol{x}_{i}, \boldsymbol{p}_{i}^{A}, \boldsymbol{p}^{J}\right)^{-1}\right\}
\end{aligned}
$$

Proof: Since power control game $\mathcal{G}$ is a two-player zero-sum game and $u_{A}\left(\boldsymbol{p}^{A}, \boldsymbol{p}^{J}\right)$ in (15) is a concave function of $\boldsymbol{p}^{A}$ and $u_{J}\left(\boldsymbol{p}^{A}, \boldsymbol{p}^{J}\right)$ in (16) is a linear (and concave) function of $\boldsymbol{p}^{J}$, the following equality holds by von Neumann's Minimax Theorem [37], [39]:

$$
\begin{aligned}
& \min _{\boldsymbol{p}^{A} \in S_{A}} \max _{\boldsymbol{p}^{J} \in S_{J}} \frac{1}{N_{T}} \sum_{i=1}^{N_{T}} \operatorname{tr}\left\{\boldsymbol{J}_{i}\left(\boldsymbol{x}_{i}, \boldsymbol{p}_{i}^{A}, \boldsymbol{p}^{J}\right)^{-1}\right\} \\
= & \max _{\boldsymbol{p}^{J} \in S_{J}} \min _{\boldsymbol{p}^{A} \in S_{A}} \frac{1}{N_{T}} \sum_{i=1}^{N_{T}} \operatorname{tr}\left\{\boldsymbol{J}_{i}\left(\boldsymbol{x}_{i}, \boldsymbol{p}_{i}^{A}, \boldsymbol{p}^{J}\right)^{-1}\right\} .
\end{aligned}
$$

In addition, $\boldsymbol{p}^{\star}=\left(\boldsymbol{p}_{\star}^{A}, \boldsymbol{p}_{\star}^{J}\right)$ satisfying the equality in (23) is a Nash equilibrium of power control game $\mathcal{G}$.

Proposition 1 states that power control game $\mathcal{G}$ admits at least one Nash equilibrium in pure strategies. In order to further analyze the equilibrium in power control game $\mathcal{G}$, the uniqueness of the Nash equilibrium is investigated in the consideration of pure strategies. The following proposition provides a sufficient condition for the uniqueness of the pure strategy Nash equilibrium.
Proposition 3: Suppose that the Fisher information matrix in (6) is positive definite. ${ }^{2}$ Then, power control game $\mathcal{G}$ has a unique Nash equilibrium in pure strategies if all the elements of $\boldsymbol{w} \triangleq \sum_{i=1}^{N_{T}} r_{i} \boldsymbol{a}_{i}^{T}$ are different, where $r_{i}$ is defined as

$$
r_{i} \triangleq \operatorname{tr}\left\{\left[\sum_{j \in \mathcal{A}_{i}^{L}} P_{i j}^{A} \lambda_{i j} \phi_{i j} \phi_{i j}^{T}\right]^{-1}\right\} .
$$

Proof: In order to prove that the Nash equilibrium of power control game $\mathcal{G}$ is unique when the condition in Proposition 3 is satisfied, it is first shown that $u_{A}\left(\boldsymbol{p}^{A}, \boldsymbol{p}^{J}\right)$ in (15) is a strictly concave function of $\boldsymbol{p}^{A}$ for a fixed $\boldsymbol{p}^{J}$. To that aim, choose arbitrary $\tilde{\boldsymbol{p}}^{A} \in S_{A}$ and $\overline{\boldsymbol{p}}^{A} \in S_{A}$ with $\tilde{\boldsymbol{p}}^{A} \neq \overline{\boldsymbol{p}}^{A}$. Then, the following relations can be obtained for any $\alpha \in(0,1)$ :

$$
\begin{aligned}
& u_{A}\left(\alpha \tilde{\boldsymbol{p}}^{A}+(1-\alpha) \overline{\boldsymbol{p}}^{A}, \boldsymbol{p}^{J}\right) \\
& =-\frac{1}{N_{T}} \sum_{i=1}^{N_{T}} \operatorname{tr}\left\{\boldsymbol{J}_{i}\left(\boldsymbol{x}_{i}, \alpha \tilde{\boldsymbol{p}}_{i}^{A}+(1-\alpha) \overline{\boldsymbol{p}}_{i}^{A}, \boldsymbol{p}^{J}\right)^{-1}\right\} \\
& =-\frac{1}{N_{T}} \sum_{i=1}^{N_{T}} \operatorname{tr}\left\{\left[\sum_{j \in \mathcal{A}_{i}^{L}} \frac{\left(\alpha \tilde{P}_{i j}^{A}+(1-\alpha) \bar{P}_{i j}\right) \lambda_{i j}}{N_{0} / 2+\boldsymbol{a}_{i}^{T} \boldsymbol{p}^{J}} \boldsymbol{\phi}_{i j} \boldsymbol{\phi}_{i j}^{T}\right]^{-1}\right\} \\
& =-\frac{1}{N_{T}} \sum_{i=1}^{N_{T}} \operatorname{tr}\left\{\left[\alpha \sum_{j \in \mathcal{A}_{i}^{L}} \frac{\tilde{P}_{i j}^{A} \lambda_{i j}}{N_{0} / 2+\boldsymbol{a}_{i}^{T} \boldsymbol{p}^{J}} \boldsymbol{\phi}_{i j} \boldsymbol{\phi}_{i j}^{T}\right.\right. \\
& \left.\left.+(1-\alpha) \sum_{j \in \mathcal{A}_{i}^{L}} \frac{\bar{P}_{i j}^{A} \lambda_{i j}}{N_{0} / 2+\boldsymbol{a}_{i}^{T} \boldsymbol{p}^{J}} \boldsymbol{\phi}_{i j} \boldsymbol{\phi}_{i j}^{T}\right]^{-1}\right\} \\
& >-\frac{1}{N_{T}} \sum_{i=1}^{N_{T}} \alpha \operatorname{tr}\left\{\left[\sum_{j \in \mathcal{A}_{i}^{L}} \frac{\tilde{P}_{i j}^{A} \lambda_{i j}}{N_{0} / 2+\boldsymbol{a}_{i}^{T} \boldsymbol{p}^{J}} \boldsymbol{\phi}_{i j} \boldsymbol{\phi}_{i j}^{T}\right]^{-1}\right\} \\
& +(1-\alpha) \operatorname{tr}\left\{\left[\sum_{j \in \mathcal{A}_{i}^{L}} \frac{\bar{P}_{i j}^{A} \lambda_{i j}}{N_{0} / 2+\boldsymbol{a}_{i}^{T} \boldsymbol{p}^{J}} \boldsymbol{\phi}_{i j} \boldsymbol{\phi}_{i j}^{T}\right]^{-1}\right\} \\
& =\alpha u_{A}\left(\tilde{\boldsymbol{p}}^{A}, \boldsymbol{p}^{J}\right)+(1-\alpha) u_{A}\left(\overline{\boldsymbol{p}}^{A}, \boldsymbol{p}^{J}\right)
\end{aligned}
$$

where the equalities in (25) and (26) are due to the definitions in (15) and (6), respectively, and the inequality in (28) follows from the fact that $\operatorname{tr}\left\{\boldsymbol{X}^{-1}\right\}$ is a strictly convex function of $\boldsymbol{X}$ if $\boldsymbol{X}$ is a symmetric positive definite matrix [40]. It is noted that $\alpha \in(0,1), \phi_{i j} \phi_{i j}^{T}$ is a symmetric positive semidefinite matrix, and $\left(\tilde{P}_{i j}^{A} \lambda_{i j}\right) /\left(N_{0} / 2+\boldsymbol{a}_{i}^{T} \boldsymbol{p}^{J}\right)$ and $\left(\bar{P}_{i j}^{A} \lambda_{i j}\right) /\left(N_{0} / 2+\boldsymbol{a}_{i}^{T} \boldsymbol{p}^{J}\right)$ are always non-negative for all $i \in\left\{1, \ldots, N_{T}\right\}$ and $j \in \mathcal{A}_{i}^{L}$.

Based on the relations in (25)-(29), it is proved that $u_{A}\left(\boldsymbol{p}^{A}, \boldsymbol{p}^{J}\right)$ in (15) is a strictly concave function of $\boldsymbol{p}^{A}$ for a fixed $p^{J}$.

Next, it is obtained that there exists a unique maximizer of $u_{J}\left(\boldsymbol{p}^{A}, \boldsymbol{p}^{J}\right)$ in (16) for a given $\boldsymbol{p}^{A}$ when the condition in Proposition 3 is satisfied. To that aim, consider the best response

\footnotetext{
${ }^{2}$ The Fisher information matrix is always positive semidefinite by definition. The assumption in the proposition corresponds to practical scenarios with a sufficient number of anchor nodes and guarantees the invertibility of the Fisher information matrix.
} 
function of player $J$ in (20). Based on a similar approach to that in [33], the solution of the optimization problem in (20) can be expressed as

$$
p_{\mathrm{BR}}^{J}(h(j))=\min \left\{P_{T}^{J}-\sum_{l=1}^{j-1} p_{\mathrm{BR}}^{J}(h(l)), P_{\text {peak }}^{J}\right\}
$$

for $j=1, \ldots, N_{J}$, where $h(j)$ denotes the index of the $j$ th largest element of vector $\boldsymbol{w}$ defined in Proposition $3, p_{\mathrm{BR}}^{J}(h(j))$ represents the $h(j)$ th element of $p_{\mathrm{BR}}^{J}$, and $\sum_{l=1}^{0}(\cdot)$ is defined as zero. For the condition that all the elements of $\boldsymbol{w}$ are different, index vector $\boldsymbol{h} \triangleq\left[h(1) h(2) \cdots h\left(N_{J}\right)\right]$ becomes unique and consequently the solution in (30) turns into a unique maximizer of $u_{J}\left(\boldsymbol{p}^{A}, \boldsymbol{p}^{J}\right)$ for a given $\boldsymbol{p}^{A}$. Therefore, based on the properties of game $\mathcal{G}$ presented in the proof of Proposition 1 and the statements proved above, it is concluded that if the condition in Proposition 3 is satisfied, then the Nash equilibrium of power control game $\mathcal{G}$ is unique.

It is important to note that the Nash equilibrium obtained by (22) based on Proposition 2 may not be unique. However, Proposition 3 provides a sufficient condition to check that the obtained Nash equilibrium is a unique equilibrium of power control game $\mathcal{G}$. If the condition in Proposition 3 is satisfied for a given Nash equilibrium, then there exists a unique equilibrium of game $\mathcal{G}$. Otherwise, the Nash equilibrium may or may not be unique. The condition in Proposition 3 depends on various system parameters such as the power strategy and the locations of the anchor nodes, the properties of the signal transmitted from the anchor nodes, the multipath components between the anchor nodes and the target nodes, and the channel coefficients between the jammer nodes and the target nodes.

In the presence of multiple Nash equilibria, the anchor and jammer nodes may choose the desired Nash equilibrium depending on the conditions and constraints in the specific application. Although the average CRLB of the target nodes (i.e., the value of the game) is the same for all Nash equilibria based on Proposition 2, the anchor and jammer nodes may prefer one Nash equilibrium over the others for the efficient use of limited resources in the wireless localization network.

\section{Power Control Game Based on Minimum and Maximum $C R L B$}

Instead of employing the average CRLB as the performance metric, it is also possible to use the worst-case CRLBs for the anchor and jammer nodes as the performance metrics. In particular, from the viewpoint of the anchor nodes, the target node with the maximum CRLB (i.e., with the worst localization accuracy) can be considered with the aim of minimizing the maximum CRLB (so that a certain level of localization accuracy can be achieved by all the target nodes). Similarly, the jammer nodes can aim to maximize the minimum CRLB of the target nodes in order to degrade the localization performance of the system. For this setting, define a new game $\overline{\mathcal{G}}$ which has the same players and the same strategy sets for the players as $\mathcal{G}$ does, except for the utility functions. For a given action profile, the utility

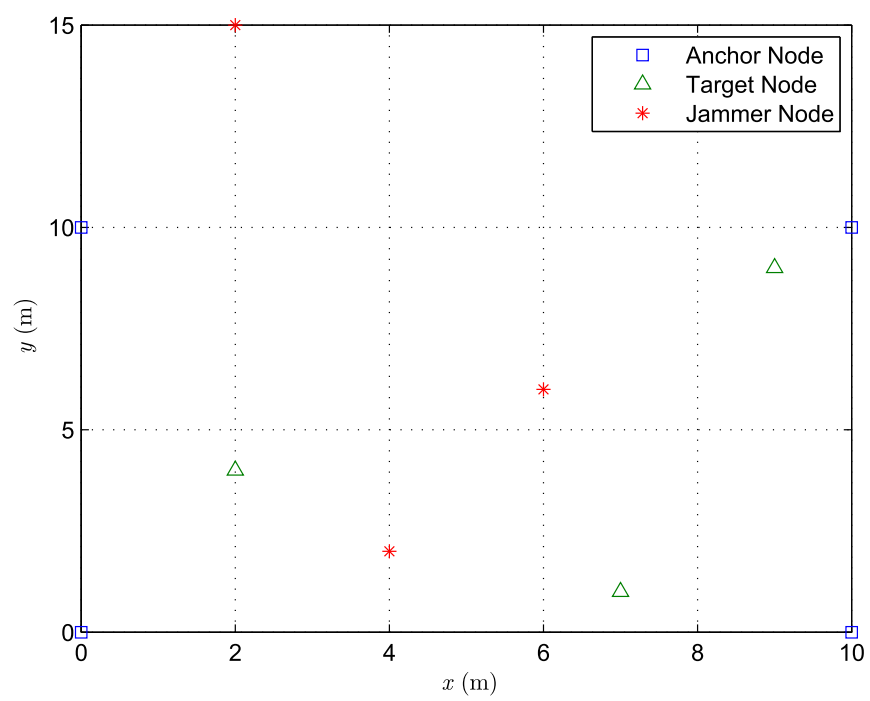

Fig. 1. Simulated network including four anchor nodes positioned at $[00]$, [10 0], [0 10], and [10 10]m., three jammer nodes positioned at [2 15], [4 2], and $\left[\begin{array}{ll}6 & 6\end{array}\right] \mathrm{m}$., and three target nodes positioned at [2 4], [7 1$]$, and [9 9$] \mathrm{m}$.

functions of player $A$ and player $J$ in game $\overline{\mathcal{G}}$ are given by

$$
\begin{aligned}
& u_{A}\left(\boldsymbol{p}^{A}, \boldsymbol{p}^{J}\right)=-\max _{i \in 1, \ldots, N_{T}} \operatorname{tr}\left\{\boldsymbol{J}_{i}\left(\boldsymbol{x}_{i}, \boldsymbol{p}_{i}^{A}, \boldsymbol{p}^{J}\right)^{-1}\right\}, \\
& u_{J}\left(\boldsymbol{p}^{A}, \boldsymbol{p}^{J}\right)=\min _{i \in 1, \ldots, N_{T}} \operatorname{tr}\left\{\boldsymbol{J}_{i}\left(\boldsymbol{x}_{i}, \boldsymbol{p}_{i}^{A}, \boldsymbol{p}^{J}\right)^{-1}\right\} .
\end{aligned}
$$

As it can be noted from the utility functions for player $A$ and player $J$ in (31) and (32), the power control game based on these utility functions is not a zero-sum game; that is, $u_{A}\left(\boldsymbol{p}^{A}, \boldsymbol{p}^{J}\right)+$ $u_{J}\left(\boldsymbol{p}^{A}, \boldsymbol{p}^{J}\right) \neq 0, \exists \boldsymbol{p}^{A} \in S_{A} \wedge \exists \boldsymbol{p}^{J} \in S_{J}$.

The utility functions in this scenario do not facilitate detailed theoretical analyses as in the case of the average CRLB based utility functions. However, the existence of a pure Nash equilibrium is still guaranteed based on the following result.

Proposition 4: There exists at least one pure Nash equilibrium in game $\overline{\mathcal{G}}$.

Proof: Game $\overline{\mathcal{G}}$ admits at least one pure Nash equilibrium if the conditions presented in the proof of Proposition 1 are satisfied. Game $\overline{\mathcal{G}}$ satisfies the first condition since game $\overline{\mathcal{G}}$ has the same strategy sets for the players as $\mathcal{G}$ does. Also, $u_{A}\left(\boldsymbol{p}^{A}, \boldsymbol{p}^{J}\right)$ in (31) and $u_{J}\left(\boldsymbol{p}^{A}, \boldsymbol{p}^{J}\right)$ in (32) are concave functions of $\boldsymbol{p}^{A}$ and $\boldsymbol{p}^{J}$, respectively, since the minimum (maximum) of concave (convex) functions is also concave (convex). Therefore, game $\overline{\mathcal{G}}$ also satisfies the second and third conditions. Consequently, based on the similar approach employed in the proof of Proposition 1, it can be shown that at least one pure-strategy Nash equilibrium exists in game $\overline{\mathcal{G}}$.

\section{NUMERICAL RESULTS}

In this section, numerical examples are provided in order to corroborate the theoretical results obtained in the previous section. To that aim, consider a wireless localization network in which four anchor nodes, three target nodes, and three jammer nodes are located as in Fig. 1. For the sake of simplicity, it is 


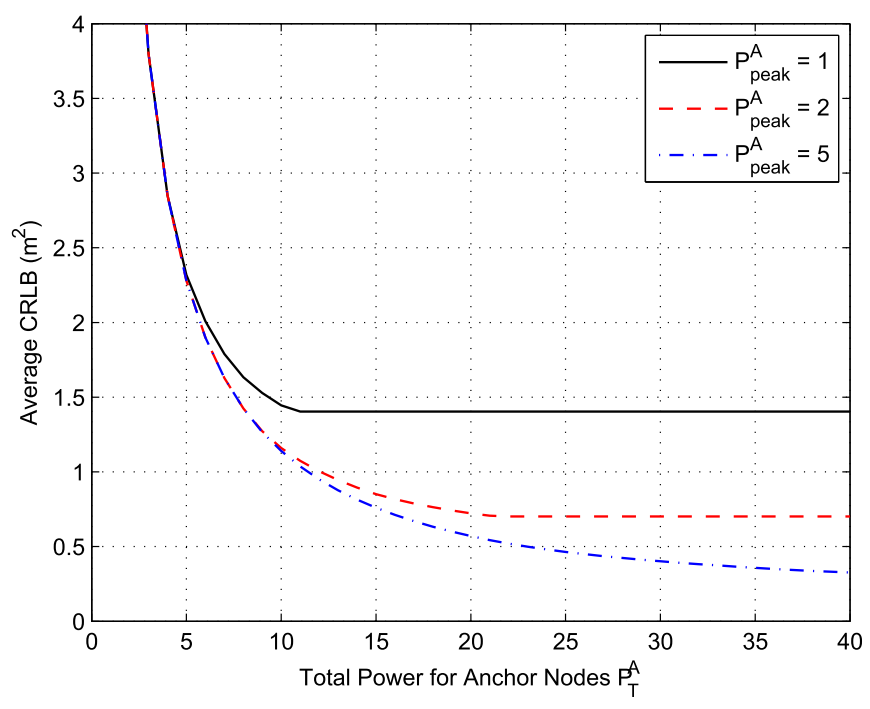

Fig. 2. Average CRLB of the target nodes versus total power of the anchor nodes for the scenario in Fig. 1 , where $P_{T}^{J}=20, P_{\text {peak }}^{J}=10$, and the anchor nodes and the jammer nodes operate at Nash equilibrium in power control game $\mathcal{G}$.

assumed that each target node has LOS connections to all of the anchor nodes. Also, the free space propagation model is considered; that is, $\lambda_{i j}$ in (7) is equal to $\lambda_{i j}=100 N_{0} \| \boldsymbol{x}_{i}-$ $\boldsymbol{y}_{j} \|^{-2} / 2$ [17]. In addition, $\left|\gamma_{i j}\right|^{2}$ is given by $\left\|\boldsymbol{x}_{i}-\boldsymbol{z}_{j}\right\|^{-2} / 2$ and $N_{0}$ is set to 2 [11].

In Fig. 2, the average CRLBs of the three target nodes (i.e., the values of the game) are plotted versus the total available power of the anchor nodes (i.e., $P_{T}^{A}$ ) for various peak powers of the anchor nodes when $P_{T}^{J}=20, P_{\text {peak }}^{J}=10$, and the anchor nodes and the jammer nodes operate at the Nash equilibrium. From the figure, it is observed that as the total power of the anchor nodes increases, the average CRLB obtained in the Nash equilibrium reduces since more strategies become available for the anchor nodes as $P_{T}^{A}$ increases. Also, it can be deduced from the figure that for lower values of the total power of the anchor nodes (e.g., $P_{T}^{A}<5$ ), the average CRLBs of the target nodes are the same for different values of $P_{\text {peak }}^{A}$ due to the dominant effect of the total power constraint on the game value. On the other hand, for higher values of the total power of the anchor nodes (e.g., $P_{T}^{A} \geq 12$ for $\left.P_{\text {peak }}^{A}=1\right)$, the average CRLB of the localization system does not change since the peak power constraint of the anchor nodes limits the use of total power available for the anchor nodes.

In order to observe the effects of the peak power constraint of the anchor nodes on the average CRLB of the target nodes, the average CRLBs of the target nodes are plotted in Fig. 3 versus the peak power of the anchor nodes for various values of the total power of the anchor nodes when $P_{T}^{J}=20$ and $P_{\text {peak }}^{J}=10$. From Fig. 3, similar observations to those for Fig. 2 are obtained. It is also stated that the average CRLBs for different values of the total power of the anchor nodes are the same when the peak power of the anchor nodes is below a certain value since the peak power constraint of the anchor nodes becomes more dominant than the total power constraint in that case.

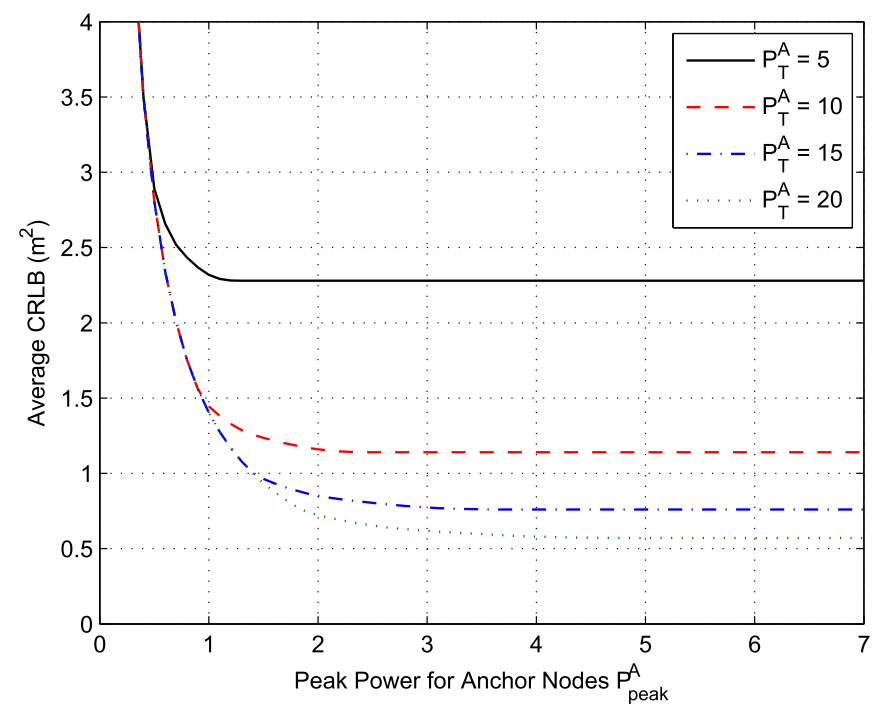

Fig. 3. Average CRLB of the target nodes versus peak power of the anchor nodes for the scenario in Fig. 1, where $P_{T}^{J}=20, P_{\text {peak }}^{J}=10$, and the anchor nodes and the jammer nodes operate at Nash equilibrium in power control game $\mathcal{G}$.

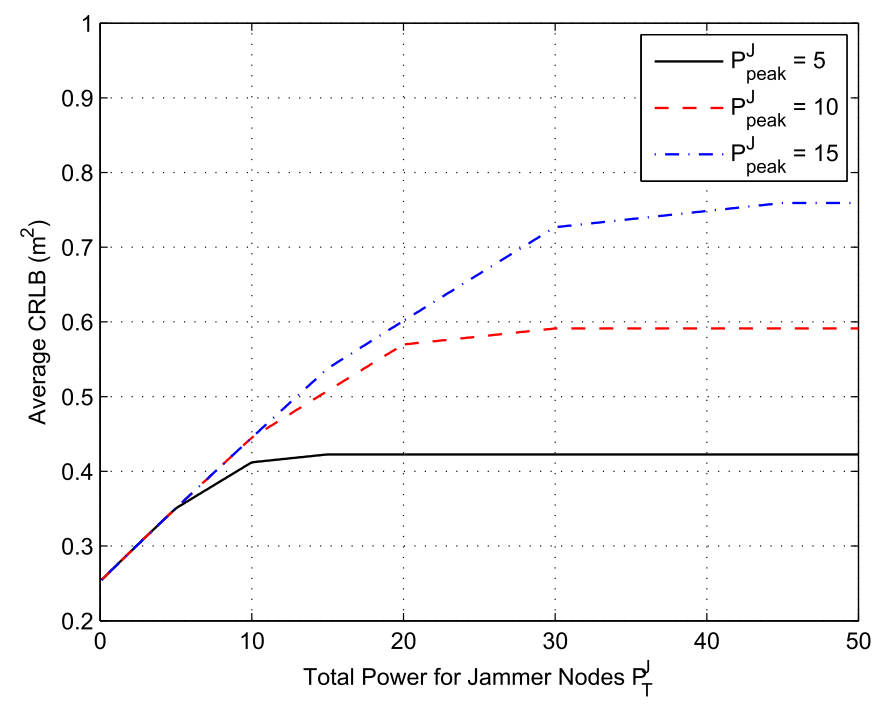

Fig. 4. Average CRLB of the target nodes versus total power of the jammer nodes for the scenario in Fig. 1 , where $P_{T}^{A}=20, P_{\text {peak }}^{A}=10$, and the anchor nodes and the jammer nodes operate at Nash equilibrium in power control game $\mathcal{G}$.

Similar to Figs. 2 and 3, the average CRLBs are plotted versus the total power of the jammer nodes for various values of the peak power and versus the peak power of the jammer nodes for different values of the total power of the jammer nodes in Figs. 4 and 5, respectively, where $P_{T}^{A}=20$ and $P_{\text {peak }}^{A}=10$. Unlike the trends in Figs. 2 and 3, the average CRLBs obtained in the Nash equilibria increase as the total power of the jammer nodes and the peak power of the jammer nodes increase in Figs. 4 and 5, respectively, since the aim of the jammer nodes is to reduce the localization performance; that is, to increase the average CRLB. Similarly, from Figs. 4 and 5, the results related 


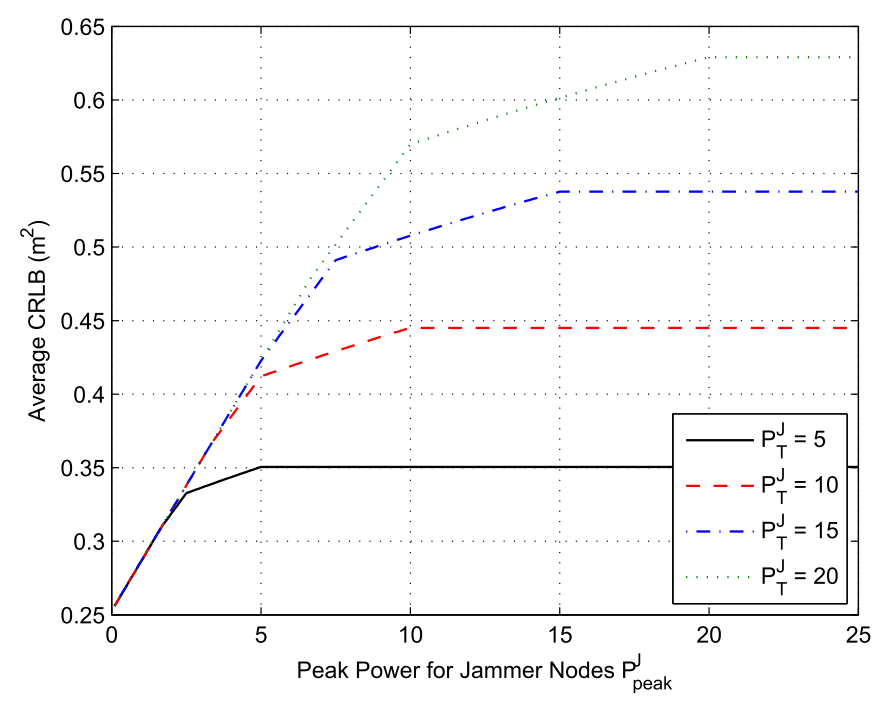

Fig. 5. Average CRLB of the target nodes versus peak power of the jammer nodes for the scenario in Fig. 1, where $P_{T}^{A}=20, P_{\text {peak }}^{A}=10$, and the anchor nodes and the jammer nodes operate at Nash equilibrium in power control game $\mathcal{G}$.

to the dominance of the constraints for different total power and peak power levels of the jammer nodes can be deduced. It is important to note that the slope of the curves in Figs. 4 and 5 changes due to the peak power and total power constraints. As an example, consider the case (i.e., the red line) in Fig. 4, where $P_{\text {peak }}^{J}=10, P_{T}^{A}=20$, and $P_{\text {peak }}^{A}=10$. The slope of the curve changes when $P_{T}^{J}=10, P_{T}^{J}=20$, and $P_{T}^{J}=30$. The reason for that can be expressed as follows: For $P_{T}^{J} \leq 10$, only one jammer node with the highest impact on the system transmits noise based on the optimization problem in (20). For $10<P_{T}^{J} \leq 20$, two jammer nodes are active in the system; that is, the jammer node with the highest impact on the system transmits noise at peak power (i.e., $P_{\text {peak }}^{J}=10$ ) whereas the other jammer node with the second highest impact on the system transmits noise such that the total power of the two nodes is equal to the total power constraint of the jammer nodes. Similarly, for $20<P_{T}^{J} \leq 30$, all the jammer nodes operate. Due to the peak power constraint (i.e., $P_{\text {peak }}^{J}=10$ for each jammer node), the power strategies of the jammer nodes remain the same for $P_{T}^{J}>30$. On the other hand, for the cases in Fig. 5, a similar process can be considered in the reverse direction. Namely, all the jammer nodes transmit noise for a lower peak power of the jammer nodes and the number of active jammer nodes in the system decreases gradually as the peak power for the jammer nodes increases.

Table I presents the Nash equilibrium strategies of the anchor and jammer nodes, which are located as in Fig. 1, for various peak power and total power constraints of the anchor and jammer nodes. It is important to note that in Table I, the Nash equilibrium strategy of the anchor nodes (i.e., player A) denoted by $\overline{\boldsymbol{p}}_{\star}^{A}$ corresponds to the reshaped version of $\boldsymbol{p}_{\star}^{A}$ in (17) and (18) for the purpose of a clear presentation. Namely, $\boldsymbol{p}^{A}$ is assumed to be defined as $\boldsymbol{p}^{A} \triangleq\left[\boldsymbol{p}_{1}^{A} \cdots \boldsymbol{p}_{N_{T}}^{A}\right]^{T}$ instead of the one in

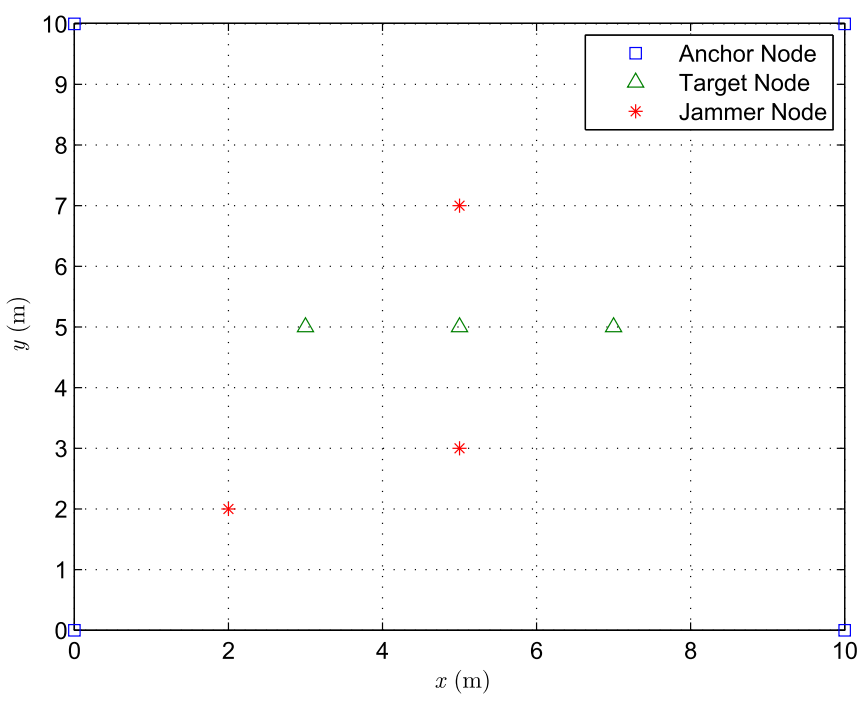

Fig. 6. Simulated network including four anchor nodes positioned at $[00]$, [10 0], [10 10], and [0 10]m., three jammer nodes positioned at [5 3], [5 7], and $\left[\begin{array}{ll}2 & 2\end{array}\right] \mathrm{m}$., and three target nodes positioned at [3 5], [5 5], and [7 5$] \mathrm{m}$.

(13). Table I provides the strategies for the anchor node and the jammer node for one Nash equilibrium obtained in each case based on the peak power and total power constraints. The results in Table I agree with Proposition 1 on that power control game $\mathcal{G}$ admits at least one pure Nash equilibrium for each case as one Nash equilibrium is provided for each case in Table I. Also, it is obtained that $u_{J}\left(\boldsymbol{p}_{\star}^{A}, \boldsymbol{p}_{\star}^{J}\right)=-u_{A}\left(\boldsymbol{p}_{\star}^{A}, \boldsymbol{p}_{\star}^{J}\right)$ for each case, as Proposition 2 states. In addition, each obtained pure Nash equilibrium in Table $\mathrm{I}$ is a unique pure Nash equilibrium based on Proposition 3 since all the elements of $\boldsymbol{w}$ presented in Table I are different in each case.

In order to investigate that power control game $\mathcal{G}$ can have multiple pure Nash equilibria for some given peak power and total power constraints, consider a wireless localization network including four anchor nodes, three target nodes, and three jammer nodes which are located as in Fig. 6. In Table II, the Nash equilibria strategies of the anchor nodes and the jammer nodes in Fig. 6 are provided for certain peak power and total power constraints. It is obtained from Table II that there exist multiple pure Nash equilibria for some peak power and total power constraints of the anchor nodes and the jammer nodes (e.g., $P_{T}^{A}=15, P_{\text {peak }}^{A}=10, P_{T}^{J}=15$, and $\left.P_{\text {peak }}^{J}=10\right)$. Also, the value of the game is unique for every Nash equilibrium as Proposition 2 states. In addition, based on Proposition 3, it can be argued that some of the elements of $\boldsymbol{w}$ provided in Table II must be the same since power control game $\mathcal{G}$ has multiple pure strategy Nash equilibria for that case, which complies with the results in Table II.

At this point, it would be useful to mention that the conventional iterative algorithm based on best response dynamics is employed in the numerical examples to obtain the Nash equilibrium. In the best response dynamics, one player chooses an arbitrary strategy first and then the other player plays the best response to the opponent's current best strategy. At each round, each player employs the best response to the current strategy of 
TABLE I

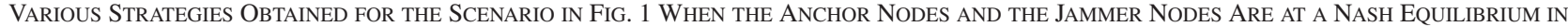
POWER CONTROL GAME $\mathcal{G}$

\begin{tabular}{|c|c|c|c|c|c|c|c|}
\hline$\left[\begin{array}{c}P_{T}^{A} \\
P_{\text {peak }}^{A} \\
P_{T}^{J} \\
P_{\text {peak }}^{J}\end{array}\right]$ & \multicolumn{4}{|c|}{$\overline{\boldsymbol{p}}_{\star}^{A}$} & $\boldsymbol{p}_{\star}^{J}$ & $\boldsymbol{w}^{T}$ & $u_{J}\left(\boldsymbol{p}_{\star}^{A}, \boldsymbol{p}_{\star}^{J}\right)\left(-u_{A}\left(\boldsymbol{p}_{\star}^{A}, \boldsymbol{p}_{\star}^{J}\right)\right)$ \\
\hline$\left[\begin{array}{l}20 \\
10 \\
20 \\
10\end{array}\right]$ & {$\left[\begin{array}{c}2.3908 \\
0 \\
0\end{array}\right.$} & $\begin{array}{l}4.2860 \\
1.6703 \\
2.5912\end{array}$ & $\begin{array}{c}0.8796 \\
0 \\
2.591\end{array}$ & $\begin{array}{cc}6 & 0 \\
& 5.0111 \\
2 & 0.5797\end{array}$ & {$\left[\begin{array}{c}0 \\
10 \\
10\end{array}\right]$} & {$\left[\begin{array}{l}0.0065 \\
0.0572 \\
0.0371\end{array}\right]$} & 0.5698 \\
\hline$\left[\begin{array}{l}10 \\
10 \\
20 \\
10\end{array}\right]$ & {$\left[\begin{array}{c}1.1954 \\
0 \\
0\end{array}\right.$} & $\begin{array}{l}2.1430 \\
0.8352 \\
1.2956\end{array}$ & $\begin{array}{c}0.4398 \\
0 \\
1.2956\end{array}$ & $\left.\begin{array}{c}0 \\
2.5056 \\
0.2898\end{array}\right]$ & {$\left[\begin{array}{c}0 \\
10 \\
10\end{array}\right]$} & {$\left[\begin{array}{l}0.0129 \\
0.1145 \\
0.0743\end{array}\right]$} & 1.1396 \\
\hline$\left[\begin{array}{l}20 \\
10 \\
10 \\
10\end{array}\right]$ & {$\left[\begin{array}{c}2.4470 \\
0 \\
0\end{array}\right.$} & $\begin{array}{l}4.3868 \\
1.7309 \\
2.4024\end{array}$ & $\begin{array}{c}0.9002 \\
0 \\
2.4024\end{array}$ & $\left.\begin{array}{c}0 \\
5.1928 \\
0.5375\end{array}\right]$ & {$\left[\begin{array}{c}0 \\
10 \\
0\end{array}\right]$} & {$\left[\begin{array}{l}0.0066 \\
0.0560 \\
0.0378\end{array}\right]$} & 0.4450 \\
\hline$\left[\begin{array}{c}20 \\
1 \\
20 \\
10\end{array}\right]$ & & {$\left[\begin{array}{ll}1 & 1 \\
1 & 1 \\
1 & 1\end{array}\right.$} & $\left.\begin{array}{ll}1 & 1 \\
1 & 1 \\
1 & 1\end{array}\right]$ & & {$\left[\begin{array}{c}0 \\
10 \\
10\end{array}\right]$} & {$\left[\begin{array}{l}0.0155 \\
0.1420 \\
0.0905\end{array}\right]$} & 1.4031 \\
\hline$\left[\begin{array}{c}20 \\
10 \\
20 \\
6\end{array}\right]$ & {$\left[\begin{array}{c}2.3341 \\
0 \\
0\end{array}\right.$} & $\begin{array}{l}4.1844 \\
1.6473 \\
2.7133\end{array}$ & $\begin{array}{c}0.8586 \\
0 \\
2.7133\end{array}$ & $\left.\begin{array}{c}0 \\
4.9420 \\
0.6070\end{array}\right]$ & {$\left[\begin{array}{l}6 \\
6 \\
6\end{array}\right]$} & {$\left[\begin{array}{l}0.0064 \\
0.0581 \\
0.0368\end{array}\right]$} & 0.4564 \\
\hline
\end{tabular}

TABLE II

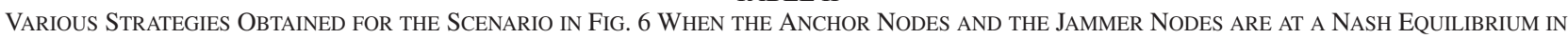
Power CONTROL Game $\mathcal{G}$

\begin{tabular}{|c|c|c|c|c|c|c|c|}
\hline$\left[\begin{array}{c}P_{T}^{A} \\
P_{\text {peak }}^{A} \\
P_{T}^{J} \\
P_{\text {peak }}^{J}\end{array}\right]$ & \multicolumn{4}{|c|}{$\overline{\boldsymbol{p}}_{\star}^{A}$} & $\boldsymbol{p}_{\star}^{J}$ & $\boldsymbol{w}^{T}$ & $u_{J}\left(\boldsymbol{p}_{\star}^{A}, \boldsymbol{p}_{\star}^{J}\right)\left(-u_{A}\left(\boldsymbol{p}_{\star}^{A}, \boldsymbol{p}_{\star}^{J}\right)\right)$ \\
\hline$\left[\begin{array}{l}15 \\
10 \\
15 \\
10\end{array}\right]$ & {$\left[\begin{array}{c}2.222 \\
1.528 \\
0\end{array}\right.$} & $\begin{array}{c}0 \\
1.5280 \\
2.2220\end{array}$ & $\begin{array}{c}0 \\
1.5280 \\
2.2220\end{array}$ & $\left.\begin{array}{cc}2.2220 \\
0 & 1.5280 \\
0 & 0\end{array}\right]$ & {$\left[\begin{array}{c}10 \\
5 \\
0\end{array}\right]$} & {$\left[\begin{array}{l}0.1801 \\
0.1801 \\
0.0690\end{array}\right]$} & 1.2715 \\
\hline$\left[\begin{array}{l}15 \\
10 \\
15 \\
10\end{array}\right]$ & {$\left[\begin{array}{c}2.222 \\
1.528 \\
0\end{array}\right.$} & $\begin{array}{c}0 \\
1.5280 \\
2.2220\end{array}$ & $\begin{array}{c}0 \\
1.5280 \\
2.2220\end{array}$ & $\left.\begin{array}{cc}2.2220 \\
0 & 1.5280 \\
0 & 0\end{array}\right]$ & {$\left[\begin{array}{c}7.5 \\
7.5 \\
0\end{array}\right]$} & {$\left[\begin{array}{l}0.1801 \\
0.1801 \\
0.0690\end{array}\right]$} & 1.2715 \\
\hline$\left[\begin{array}{l}15 \\
10 \\
15 \\
10\end{array}\right]$ & {$\left[\begin{array}{c}2.222 \\
1.528 \\
0\end{array}\right.$} & $\begin{array}{c}0 \\
1.5280 \\
2.2220\end{array}$ & $\begin{array}{c}0 \\
1.5280 \\
2.2220\end{array}$ & $\left.\begin{array}{cc}2.2220 \\
0 & 1.5280 \\
0 & 0\end{array}\right]$ & {$\left[\begin{array}{c}5 \\
10 \\
0\end{array}\right]$} & {$\left[\begin{array}{l}0.1801 \\
0.1801 \\
0.0690\end{array}\right]$} & 1.2715 \\
\hline
\end{tabular}

the opponent iteratively and the algorithm terminates when no players have an incentive to deviate from their previous strategies, which corresponds to a Nash equilibrium in the game. When the condition in Proposition 3 is satisfied, the obtained Nash equilibrium is guaranteed to be unique. On the other hand, when that condition is not satisfied, that is, when some elements of $\boldsymbol{w}$ are identical, the power levels of the corresponding jammer nodes can be redistributed and the resulting strategies for the anchor and jammer nodes are checked to determine if another Nash equilibrium is achieved. In order to verify that the resulting strategies constitute a different Nash equilibrium, the best response strategy of the anchor nodes to the resulting strategy of the jammer nodes is determined first based on the best response function of the anchor nodes in (19). Then, if the obtained 


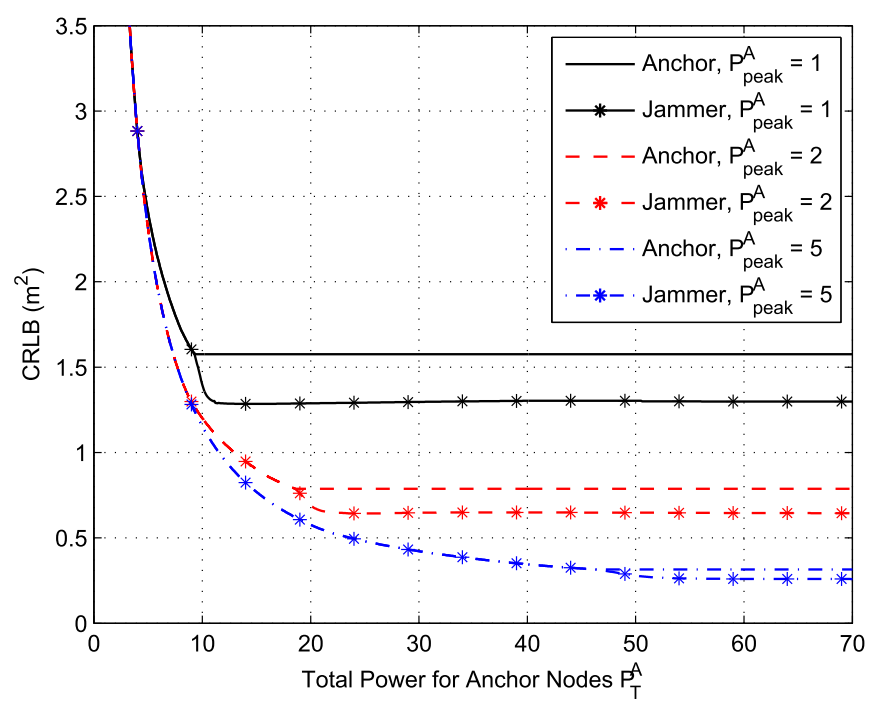

Fig. 7. Minimum and maximum CRLBs (i.e., absolute utility values for the jammer and anchor nodes, respectively) of the target nodes versus total power of the anchor nodes for the scenario in Fig. 1, where $P_{T}^{J}=20, P_{\text {peak }}^{J}=10$, and the anchor nodes and the jammer nodes operate at Nash equilibrium in power control game $\overline{\mathcal{G}}$.

strategy of the anchor nodes does not differ from the strategy of the anchor nodes in the previous Nash equilibrium, it is concluded that the resulting strategies for the anchor and jammer nodes obtained by redistributing the power levels of the jammer nodes correspond to another Nash equilibrium. Otherwise, if the strategies of the anchor nodes do not match, the resulting strategies cannot be considered as a Nash equilibrium and other possible strategies of the jammer nodes produced based on redistribution of the power levels may be examined to find another Nash equilibrium. In this way, multiple Nash equilibria can be obtained, as in Table II.

To analyze power control game $\overline{\mathcal{G}}$ in which the utility functions of the players are based on the minimum and maximum CRLBs instead of the average CRLB (see Section III-D), consider the wireless localization network in Fig. 1. In Fig. 7, the minimum and maximum CRLBs of the target nodes are plotted versus the total available power of the anchor nodes for various values of the peak power constraint of the anchor nodes when $P_{T}^{J}=20$ and $P_{\text {peak }}^{J}=10$. It is noted that for low values of the total power constraint of the anchor nodes, the utility functions of the anchor nodes and the jammer nodes become equal in magnitude; that is, the sum of the utility functions of the players is equal to zero. On the other hand, the utility functions of the anchor nodes and the jammer nodes are not equal for higher values of the total power constraint of the anchor nodes. Then, in Fig. 8, the minimum and maximum CRLBs of the target nodes are plotted versus the peak power of the anchor nodes when the anchor nodes and the jammer nodes operate at the Nash equilibrium, $P_{T}^{J}=20$, and $P_{\text {peak }}^{J}=10$. Unlike the previous figure, the utility functions of the players in game $\overline{\mathcal{G}}$ differ in magnitude for low values of the peak power of the anchor nodes. On the other hand, for high values of the peak power of the anchor nodes, the sum of the utility functions of the anchor and jammer nodes becomes

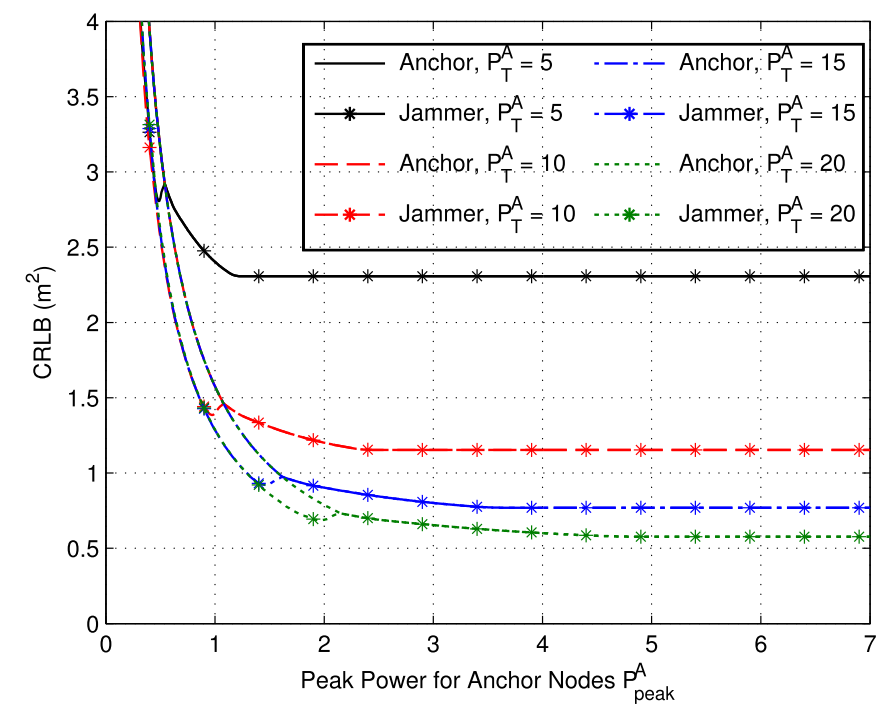

Fig. 8. Minimum and maximum CRLBs (i.e., absolute utility values for the jammer and anchor nodes, respectively) of the target nodes versus peak power of the anchor nodes for the scenario in Fig. 1 , where $P_{T}^{J}=20, P_{\text {peak }}^{J}=10$, and the anchor nodes and the jammer nodes operate at Nash equilibrium in power control game $\overline{\mathcal{G}}$.

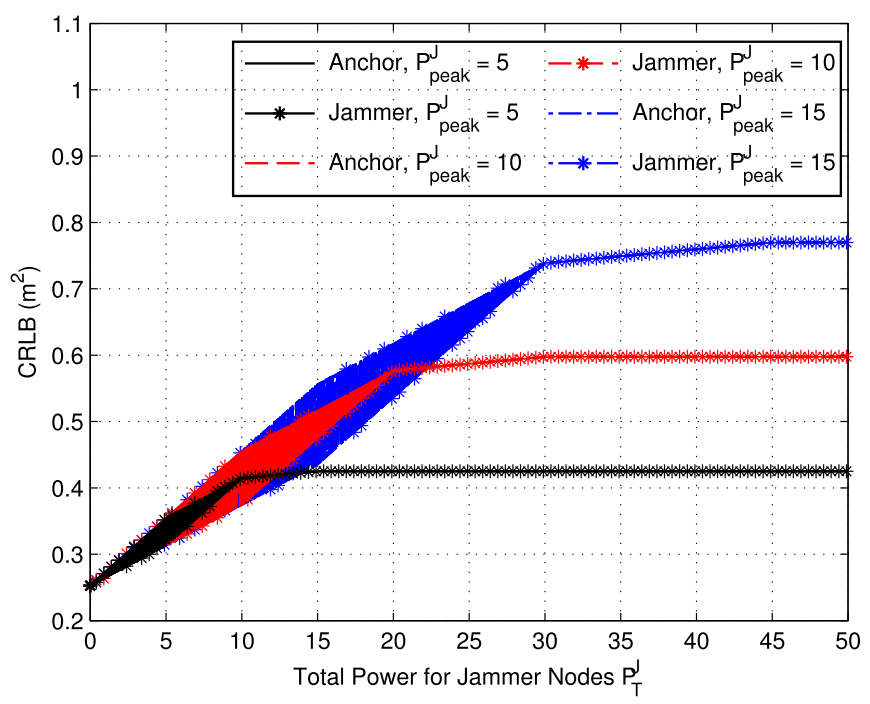

Fig. 9. Minimum and maximum CRLBs (i.e., absolute utility values for the jammer and anchor nodes, respectively) of the target nodes versus total power of the jammer nodes for the scenario in Fig. 1 , where $P_{T}^{A}=20, P_{\text {peak }}^{A}=10$, and the anchor nodes and the jammer nodes operate at Nash equilibrium in power control game $\overline{\mathcal{G}}$.

zero. It is also important to emphasize that as the total power of the anchor nodes increases, the CRLBs (i.e., the minimum of targets' CRLBs for the jammer nodes and the maximum of targets' CRLBs for the anchor nodes) obtained in the Nash equilibrium reduce. Similar plots to those in Figs. 7 and 8 are presented in Figs. 9 and 10 for the jammer nodes considering various values of the total power and peak power constraints of the jammer nodes when $P_{T}^{A}=20$ and $P_{\text {peak }}^{A}=10$. From Figs. 9 and 10, it is noticed that multiple Nash equilibria can be observed for power control game $\overline{\mathcal{G}}$ in some cases and the magnitude of the utilities 


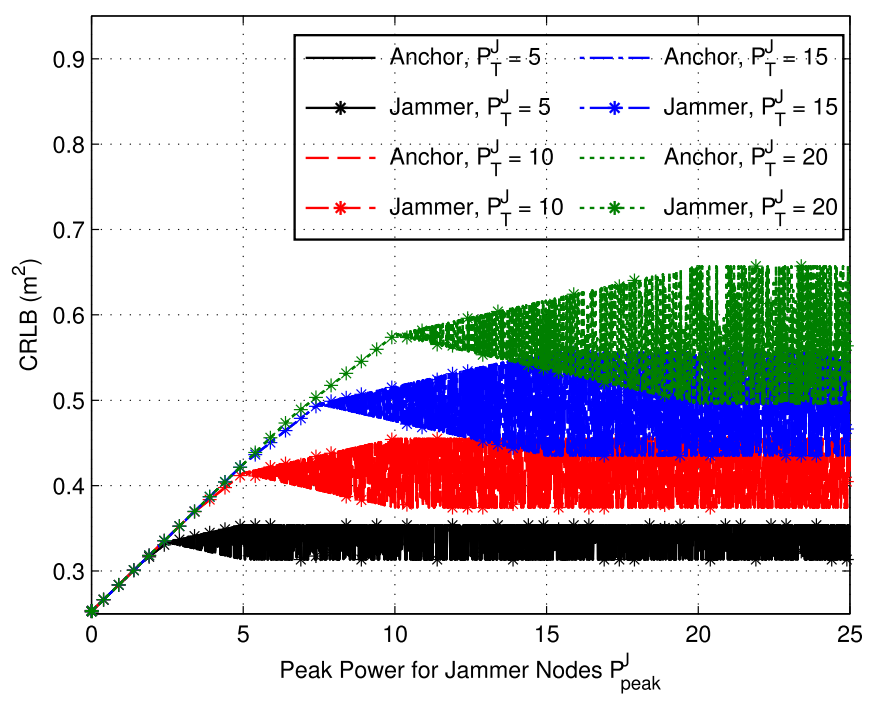

Fig. 10. Minimum and maximum CRLBs (i.e., absolute utility values for the jammer and anchor nodes, respectively) of the target nodes versus peak power of the jammer nodes for the scenario in Fig. 1, where $P_{T}^{A}=20, P_{\mathrm{peak}}^{A}=10$, and the anchor nodes and the jammer nodes operate at Nash equilibrium in power control game $\overline{\mathcal{G}}$.

obtained in those Nash equilibria points can get the values represented in the shaded regions of Figs. 9 and 10. However, for some values of the constraints, the Nash equilibria may be unique (e.g., for high values of the total power of the jammer nodes). Lastly, the results in the figures comply with the statement in Proposition 4 that power control game $\overline{\mathcal{G}}$ has at least one pure Nash equilibrium.

\section{CONCLUDING REMARKS}

In this paper, interactions between anchor and jammer nodes have been analyzed for a wireless localization network. Based on a game-theoretic framework, two types of power control games between anchor and jammer nodes have been investigated by employing the average CRLB and the worst-case CRLBs of the target nodes (from the viewpoints of the anchor and jammer nodes) as performance metrics. It has been proved that both games have at least one pure strategy Nash equilibrium. This implies that there exist deterministic power allocation strategies for the anchor and jammer nodes that lead to one or more Nash equilibria in both games. In addition, an approach has been presented in order to figure out the Nash equilibrium of the game which employs the average CRLB as the performance metric, and a sufficient condition has been provided to determine the uniqueness of the Nash equilibrium. The theoretical investigations have been illustrated via numerical examples. As an interesting direction for future work, uncertainty on various parameters of anchor and jammer nodes can be incorporated into the game models, and different game models such as stochastic and repetitive games can be considered for the localization performance of target nodes in the presence of jammer nodes in a wireless localization network.

\section{REFERENCES}

[1] Z. Sahinoglu, S. Gezici, and I. Gvenc, Ultra-Wideband Positioning Systems: Theoretical Limits, Ranging Algorithms, and Protocols. New York, NY, USA: Cambridge Univ. Press, 2008.

[2] R. Zekavat and R. M. Buehrer, Handbook of Position Location: Theory, Practice and Advances, 1st ed. New York, NY, USA: Wiley-IEEE Press, 2011.

[3] S. Gezici, "A survey on wireless position estimation," Wireless Personal Commun., vol. 44, no. 3, pp. 263-282, Feb. 2008.

[4] Y. Shen and M. Win, "Fundamental limits of wideband localizationPart I: A general framework," IEEE Trans. Inf. Theory, vol. 56, no. 10, pp. 4956-4980, Oct. 2010.

[5] G. Zanca, F. Zorzi, A. Zanella, and M. Zorzi, "Experimental comparison of RSSI-based localization algorithms for indoor wireless sensor networks," in Proc. Workshop Real-World Wireless Sensor Netw., Glasgow, U.K., Apr. 2008, pp. 1-5.

[6] D. Dardari, C. C. Chong, and M. Win, "Threshold-based time-of-arrival estimators in UWB dense multipath channels," IEEE Trans. Commun., vol. 56, no. 8, pp. 1366-1378, Aug. 2008.

[7] L. Mailaender, "On the geolocation bounds for round-trip time-of-arrival and all non-line-of-sight channels," EURASIP J. Adv. Signal Process., vol. 2008, no. 1, pp. 1-10, 2007.

[8] T. S. Rappaport, J. H. Reed, and B. D. Woerner, "Position location using wireless communications on highways of the future," IEEE Commun. Mag., vol. 34, no. 10, pp. 33-41, Oct. 1996.

[9] D. Niculescu and B. Nath, "Ad hoc positioning system (APS) using AOA," in Proc. IEEE Conf. Comput. Commun., Mar. 2003, vol. 3, pp. 1734-1743.

[10] S. Gezici et al., "Localization via ultra-wideband radios: A look at positioning aspects for future sensor networks," IEEE Signal Process. Mag., vol. 22, no. 4, pp. 70-84, Jul. 2005.

[11] S. Gezici, M. R. Gholami, S. Bayram, and M. Jansson, "Jamming of wireless localization systems," IEEE Trans. Commun., vol. 64, no. 6, pp. 2660-2676, Jun. 2016.

[12] H. Godrich, A. P. Petropulu, and H. V. Poor, "Power allocation strategies for target localization in distributed multiple-radar architectures," IEEE Trans. Signal Process., vol. 59, no. 7, pp. 3226-3240, Jul. 2011.

[13] J. Chen, W. Dai, Y. Shen, V. K. N. Lau, and M. Z. Win, "Power management for cooperative localization: A game theoretical approach," IEEE Trans. Signal Process., vol. 64, no. 24, pp. 6517-6532, Dec. 2016.

[14] H. Hu and N. Wei, "A study of GPS jamming and anti-jamming," in Proc. 2nd Int. Conf. Power Electron. Intell. Transport. Syst., Dec. 2009, vol. 1, pp. 388-391.

[15] D. Lu, R. Wu, and H. Liu, "Global positioning system anti-jamming algorithm based on period repetitive CLEAN," IET Radar, Sonar, Navig., vol. 7, no. 2, pp. 164-169, Feb. 2013.

[16] Y. Shen and M. Z. Win, "Energy efficient location-aware networks," in Proc. IEEE Int. Conf. Commun., May 2008, pp. 2995-3001.

[17] W. W.-L. Li, Y. Shen, Y. J. Zhang, and M. Z. Win, "Robust power allocation for energy-efficient location-aware networks," IEEE/ACM Trans. Netw., vol. 21, no. 6, pp. 1918-1930, Dec. 2013.

[18] T. Wang, G. Leus, and L. Huang, "Ranging energy optimization for robust sensor positioning based on semidefinite programming," IEEE Trans. Signal Process., vol. 57, no. 12, pp. 4777-4787, Dec. 2009.

[19] T. Wang and G. Leus, "Ranging energy optimization for robust sensor positioning with collaborative anchors," in Proc. IEEE Int. Conf. Acoust., Speech, Signal Process., Mar. 2010, pp. 2714-2717.

[20] R. El-Bardan, S. Brahma, and P. K. Varshney, "Strategic power allocation with incomplete information in the presence of a jammer," IEEE Trans. Commun., vol. 64, no. 8, pp. 3467-3479, Aug. 2016.

[21] V. S. S. Nadendla, V. Sharma, and P. K. Varshney, "On strategic multiantenna jamming in centralized detection networks," IEEE Signal Process. Lett., vol. 24, no. 2, pp. 186-190, Feb. 2017.

[22] J. Chen, W. Dai, Y. Shen, V. K. N. Lau, and M. Z. Win, "Resource management games for distributed network localization," IEEE J. Sel. Areas Commun., vol. 35, no. 2, pp. 317-329, Feb. 2017.

[23] M. K. Simon, J. K. Omura, R. A. Scholtz, and B. K. Levitt, Spread Spectrum Communications. Rockville, MD, USA: Comput. Sci. Press, 1985.

[24] M. Weiss and S. C. Schwartz, "On optimal minimax jamming and detection of radar signals," IEEE Trans. Aerosp. Electron. Syst., vol. AES-21, no. 3, pp. 385-393, May 1985.

[25] R. J. McEliece and W. E. Stark, "An information theoretic study of communication in the presence of jamming," in Proc. Int. Conf. Commun., 1981, vol. 3, pp. 45.3.1-45.3.5. 
[26] I. Shomorony and A. S. Avestimehr, "Worst-case additive noise in wireless networks," IEEE Trans. Inf. Theory, vol. 59, no. 6, pp. 3833-3847, Jun. 2013.

[27] T. M. Cover and J. A. Thomas, Elements of Information Theory. New York, NY, USA: Wiley-Interscience, 1991.

[28] A. Lapidoth, "Nearest neighbor decoding for additive non-Gaussian noise channels," IEEE Trans. Inf. Theory, vol. 42, no. 5, pp. 1520-1529, Sep. 1996.

[29] S. M. Kay, Fundamentals of Statistical Signal Processing: Estimation Theory. Upper Saddle River, NJ, USA: Prentice-Hall, 1993.

[30] Y. Qi, H. Kobayashi, and H. Suda, "Analysis of wireless geolocation in a non-line-of-sight environment," IEEE Trans. Wireless Commun., vol. 5, no. 3, pp. 672-681, Mar. 2006.

[31] A. Mallat, S. Gezici, D. Dardari, C. Craeye, and L. Vandendorpe, "Statistics of the MLE and approximate upper and lower bounds-Part I: Application to TOA estimation," IEEE Trans. Signal Process., vol. 62, no. 21, pp. 5663-5676, Nov. 2014.

[32] D. Dardari and M. Z. Win, "Ziv-Zakai bound on time-of-arrival estimation with statistical channel knowledge at the receiver," in Proc. IEEE Int. Conf. Ultra-Wideband, Sep. 2009, pp. 624-629.

[33] S. Gezici, S. Bayram, M. N. Kurt, and M. R. Gholami, "Optimal jammer placement in wireless localization systems," IEEE Trans. Signal Process., vol. 64, no. 17, pp. 4534-4549, Sep. 2016.

[34] J. F. Nash, "Non-cooperative games," Ann. Math., vol. 54, pp. 289-295, 1951.

[35] Z. Han, Game Theory in Wireless and Communication Networks: Theory, Models, and Applications. Cambridge, U.K.: Cambridge Univ. Press, 2012.

[36] W. W. L. Li, Y. Shen, Y. J. Zhang, and M. Z. Win, "Robust power allocation for energy-efficient location-aware networks," IEEE/ACM Trans. Netw., vol. 21, no. 6, pp. 1918-1930, Dec. 2013.

[37] A. Washburn, Two-Person Zero-Sum Games International Series in Operations Research \& Management Science. New York, NY, USA: Springer, 2013.

[38] S. Boyd and L. Vandenberghe, Convex Optimization. Cambridge, U.K.: Cambridge Univ. Press, 2004.

[39] J. v. Neumann, "Zur theorie der gesellschaftsspiele," Mathematische Annalen, vol. 100, pp. 295-320, 1928.

[40] A. Ghosh, S. Boyd, and A. Saberi, "Minimizing effective resistance of a graph," SIAM Rev., vol. 50, no. 1, pp. 37-66, 2008.

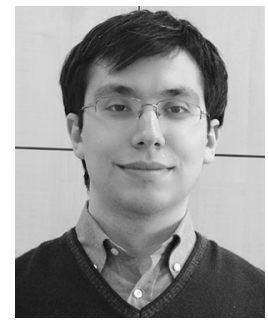

Ahmet Dundar Sezer was born in Emet, Kutahya, Turkey, in 1989. He received both the B.S. and M.S. degrees in electrical and electronics engineering from Bilkent University, Ankara, Turkey, in 2011 and 2013 , respectively. He is currently working toward the Ph.D. degree with Bilkent University. His current research interests include signal processing, wireless communications, and optimization.

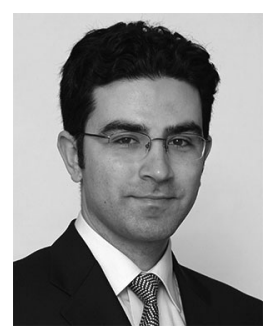

Sinan Gezici (S'03-M'06-SM'11) received the B.S. degree from Bilkent University, Ankara, Turkey, in 2001, and the Ph.D. degree in electrical engineering from Princeton University, Princeton, NJ, USA, in 2006. From 2006 to 2007, he worked at Mitsubishi Electric Research Laboratories, Cambridge, MA, USA. Since 2007, he has been with the Department of Electrical and Electronics Engineering, Bilkent University, where he is currently a Professor. His research interests include the areas of detection and estimation theory, wireless communications, and localization systems. Among his publications in these areas is the book: UltraWideband Positioning Systems: Theoretical Limits, Ranging Algorithms, and Protocols (Cambridge University Press, 2008). He was an Associate Editor for IEEE TRANSACTIONS ON COMMUNICATIONS, IEEE WiRELESS COMMUNICATIONS LETTERS, and Journal of Communications and Networks. 